\title{
Zonas del Sitio Ramsar 1602 analizadas para hidrocarburos fracción pesada en Tuxpan,
} Veracruz

Areas of Ramsar Site 1602 analyzed for heavy fraction hydrocarbons in Tuxpan, Veracruz

González-Sánchez Arianna Rubí ${ }^{1 凶}$, Elorza Martínez Pablo², Alarcón Pulido Sara Aida², Hernández Sánchez María de la Luz ${ }^{2}$, Rodríguez Betancourt Alejandra ${ }^{2}$

${ }^{1}$ Investigador independiente. ${ }^{2}$ Facultad de Ciencias Biológico Agropecuarias, Universidad Veracruzana. Campus Poza Rica-Tuxpan, carretera Tuxpan-Tampico Kilómetro 7.5, Colonia Universitaria, C.P. 92870 Tuxpan, Ver.

${ }^{凶}$ Autor para correspondencia: glezsan.ar@gmail.com

Recibido: $15 / 09 / 2018$

Aceptado: 15/11/2018

\section{RESUMEN}

El manglar es un ecosistema complejo y dinámico, considerado un humedal de importancia internacional ya que posee una fuerte interrelación inter/intra específica con el medio. Su importancia radica en innumerables servicios que brindan a distintos ámbitos, incluyendo el económico. Sin embargo, pese a esto, los manglares han sido impactados negativamente por la contaminación, teniendo una tasa anual de deforestación de aproximadamente 2.5\%, pasando de 775 mil ha en 2005 a 764 mil 486 ha en 2010. El objetivo de esta investigación fue establecer dos zonas del sitio Ramsar 1602 "Manglares y Humedales de Tuxpan", una al norte y otra al sur (ZN y ZS), de una hectárea de superficie cada una. Dentro de los principales contaminantes del área se encuentran efectos del turismo, infraestructura carretera y petrolera, actividades agropecuarias y marícolas, la reducción del flujo de agua por obras de riego y contaminación de las aguas por agroquímicos, metales pesados, nutrimentos e hidrocarburos asociados a varias actividades, principalmente en la zona norte. Se evaluaron concentraciones de hidrocarburos fracción pesada (HFP) en 16 submuetras de los dos áreas obtenidas a una profundidad de $70 \mathrm{~cm}$ de la superficie para obtener una muestra compuesta por cada zona, la cual fue analizada (EPA 1661a y 9071b con algunas modificaciones), cuantificando los HFP por diferencia de peso. Se encontraron hidrocarburos fracción pesada en ambas zonas de muestreo, con la mayor concentración en la zona norte, sin embargo, las concentraciones no fueron estadísticamente diferentes.

Palabras clave: HFP, fracción pesada, manglares, contaminación.

\begin{abstract}
The mangrove is a complex and dynamic ecosystem, considered a wetland of international importance since it has a strong inter / intra -specific interrelation with the environment. Its importance lies in
\end{abstract}


innumerable services that provide different areas, including the economic. However, despite this, mangroves have been negatively impacted by pollution, having an annual deforestation rate of approximately $2.5 \%$, from 775 thousand ha in 2005 to 764 thousand 486 ha in 2010 . The objective of this research was to establish two areas of Ramsar site 1602 "Manglares y Humedales de Tuxpan", one to the north and one to the south (ZN and ZS), each one hectare. Among the main pollutants of the area are the effects of tourism, road and oil infrastructure, agricultural and marine activities, the reduction of water flow by irrigation works and water contamination by agrochemicals, heavy metals, nutrients and hydrocarbons associated with several activities, mainly in the northern zone. Heavy hydrocarbon concentrations (HFP) were evaluated in 16 sub-samples of the two areas obtained at a depth of $70 \mathrm{~cm}$ from the surface to obtain a composite sample for each zone, which was analyzed (EPA 1661a and 9071b with some modifications), quantifying the HFP by weight difference. Heavy fraction hydrocarbons were found in both sampling areas, with the highest concentration in the northern zone, however, the concentrations were not statistically different.

Keywords: HFP, heavy fraction, mangroves, contamination.

\section{INTRODUCCIÓN}

El manglar es un ecosistema complejo formado por vegetación arbórea, fauna, flora y el medio físico sobre el que se establece con una fuerte interrelación inter/intra específica. $\mathrm{Su}$ importancia radica en los innumerables servicios que brindan en distintos ámbitos (FAO, 2010). Ayuda al control de diversas formas de contaminación, incluyendo cantidades excesivas de nitrógeno y fosfatos, productos del petróleo y compuestos halogenados. Funciona como un estabilizador y protector de las líneas costeras, zonas de cría y alimentación para numerosas especies; es sitio de anidamiento de aves costeras y migratorias, además de su gran valor económico. Sin embargo, los manglares han tenido una fuerte reducción nacional que equivale a un $65 \%$, con una tasa anual de deforestación de aproximadamente $2.5 \%$ (INE, 2005), pasando de un área de 775 mil ha en 2005 a 764 mil 486 ha en 2010 (CONABIO, 2009).

El sitio Ramsar 1602 "Manglares y Humedales de Tuxpan" es afectado principalmente por la deforestación, asentamientos humanos, efectos del turismo, infraestructura carretera y petrolera, actividades agropecuarias; reducción y contaminación de las aguas por agroquímicos, metales pesados, nutrimentos e hidrocarburos asociados a actividades propias de la zona (MorenoCasasola et al., 2002; Farrapeira et al., 2009). En los alrededores de la laguna Tampamachoco se tienen instalaciones de compañías industriales, de PEMEX y CFE, ésta última ubicada en la región norte de Tuxpan, Veracruz, y en donde se pueden encontrar zonas con alta mortalidad o gran deterioro de la comunidad de manglar; se cuenta también con una zona al sur en donde el desarrollo del manglar es más estable y la vegetación en general muestra un buen estado de conservación.

Por lo que el objetivo de esta investigación fue establecer dos zonas del sitio Ramsar 1602 "Manglares y Humedales de Tuxpan", una al norte y otra al sur (ZN y ZS). Se evaluaron concentraciones de hidrocarburos fracción pesada (HFP) en 16 submuetras de los dos áreas obtenidas a una profundidad de $70 \mathrm{~cm}$ de la superficie para obtener una muestra compuesta por cada zona, la cual fue analizada (EPA 1661 a y 9071 b con algunas 
modificaciones) (EPA 1998; 2000) cuantificando los HFP por diferencia de peso.

\section{MATERIALES Y MÉTODOS}

\section{Área de estudio}

El estudio se llevó a cabo en el sitio Ramsar 1602 ubicado entre las coordenadas $21^{\circ} 00^{\prime} \mathrm{N}$ y $097^{\circ} 21^{\prime} \mathrm{W}$ abarcando un total de 6,870 ha (Ramsar, 2014) al norte del estado de Veracruz, México. El tipo de vegetación más importante es el manglar que es alimentado por dos principales cuerpos de agua, la Laguna de y el Río (CONABIO, 2009); este último divide al manglar en dos zonas, una ubicada al norte (ZN), y la otra al sur (ZS). Predomina el clima cálido húmedo Am (f) y cálido subhúmedo $\mathrm{A}$ (w2), con una temperatura media anual entre $22^{\circ} \mathrm{C}$ y 24. $9^{\circ} \mathrm{C} \quad$ (Basáñez-Muñoz, 2005). Se seleccionaron dos zonas de muestreo, una en la ZN y otra en la ZS, se delimitó un área de una hectárea cubriendo un total de dos hectáreas. La época de muestreo fue de junio a agosto del 2014 (figura 1).

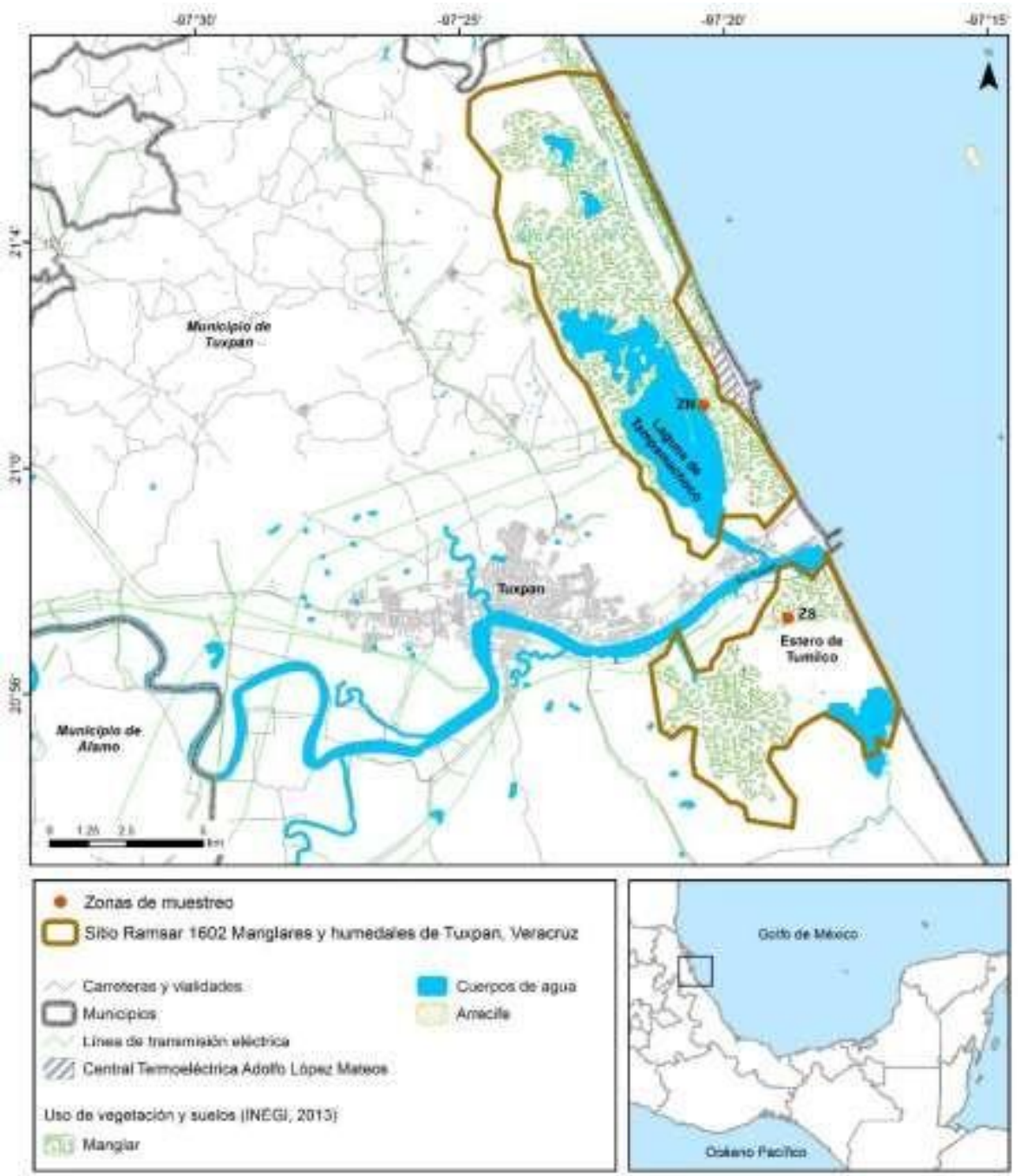

Figura 1. Ubicación de las zonas de muestreo en el sitio RAMSAR 1602; Tuxpan, México; zona norte (ZN) y zona sur (Zs). 


\section{Análisis de Hidrocarburos Fracción Pesada (HFP)}

Para determinar hidrocarburos de fracción pesada (HFP) en muestras de suelo se utilizaron los métodos EPA 1661a y 9071b (EPA, 1998; 2000) modificados; las determinaciones se hicieron por triplicado.

La cuantificación de los HFP se realizó por de diferencias de pesos $\left(\mathrm{mg}^{*} \mathrm{~kg}^{-1}\right)$ :

$$
\mathrm{HFP}=\left[\mathrm{P}_{\mathrm{M}}+\mathrm{HC}-\mathrm{P}_{\mathrm{M}}\right] / \mathrm{P}_{\mathrm{S}}
$$

\section{Dónde:}

HFP $=$ Hidrocarburos fracción pesada $\left(\mathrm{mg} \cdot \mathrm{kg}^{-1}\right)$; $\mathbf{P}_{\mathbf{M}+\mathbf{H C}}=$ Peso del matraz del Segundo proceso del filtrado $(\mathrm{mg})^{\mathbf{3}} \mathbf{P}_{\mathbf{M}}=$ Peso del matraz limpio a peso constante $(\mathrm{mg})^{2}$; $\mathbf{P}_{\mathbf{S}}=$ Peso de la muestra utilizada $(\mathrm{kg}) \mathbf{1}$

\section{Análisis de datos}

Se realizó una prueba de Shapiro Wilk para comprobación de normalidad, ANOVA y una comparación de medias de Tukey con un nivel de significancia del 0.05 , el análisis se realizó con el paquete estadístico SPSS (Versión 12.0
1S, SPSS Inc.). La representación gráfica de los datos se realizó con el programa SigmaPlot (Jandel Scientific, ver. 11).

\section{RESULTADOS}

\section{Determinación de la presencia de hidrocarburos de fracción pesada (HFP)}

La contaminación por HFP se registró en ambas zonas de estudio (norte y sur). La cercanía de la $\mathrm{ZN}$ a la contaminación por el desarrollo agrícola, urbano, turístico e industrial adyacente a la laguna de Tampamachoco la hace más vulnerable, sobrepasando con $84.8 \%$ el límite máximo permisible para uso agrícola, forestal, pecuario y de conservación (SEMARNAT, 2013). Si se considera el recorrido del viento y la distancia de $21 \mathrm{~km}$ aproximadamente, puede contemplarse la dispersión de los contaminantes en menor proporción a la ZS sobrepasando lo establecido por la NOM-138 de 3000 mg kg-1, sólo con $12.4 \%$.

La prueba estadística de Man-Whitney señaló que la diferencias en la concentración entre ambas zonas fue significativa $(U=0, Z=2.8022$; $\mathrm{p}=0.0005)$. 


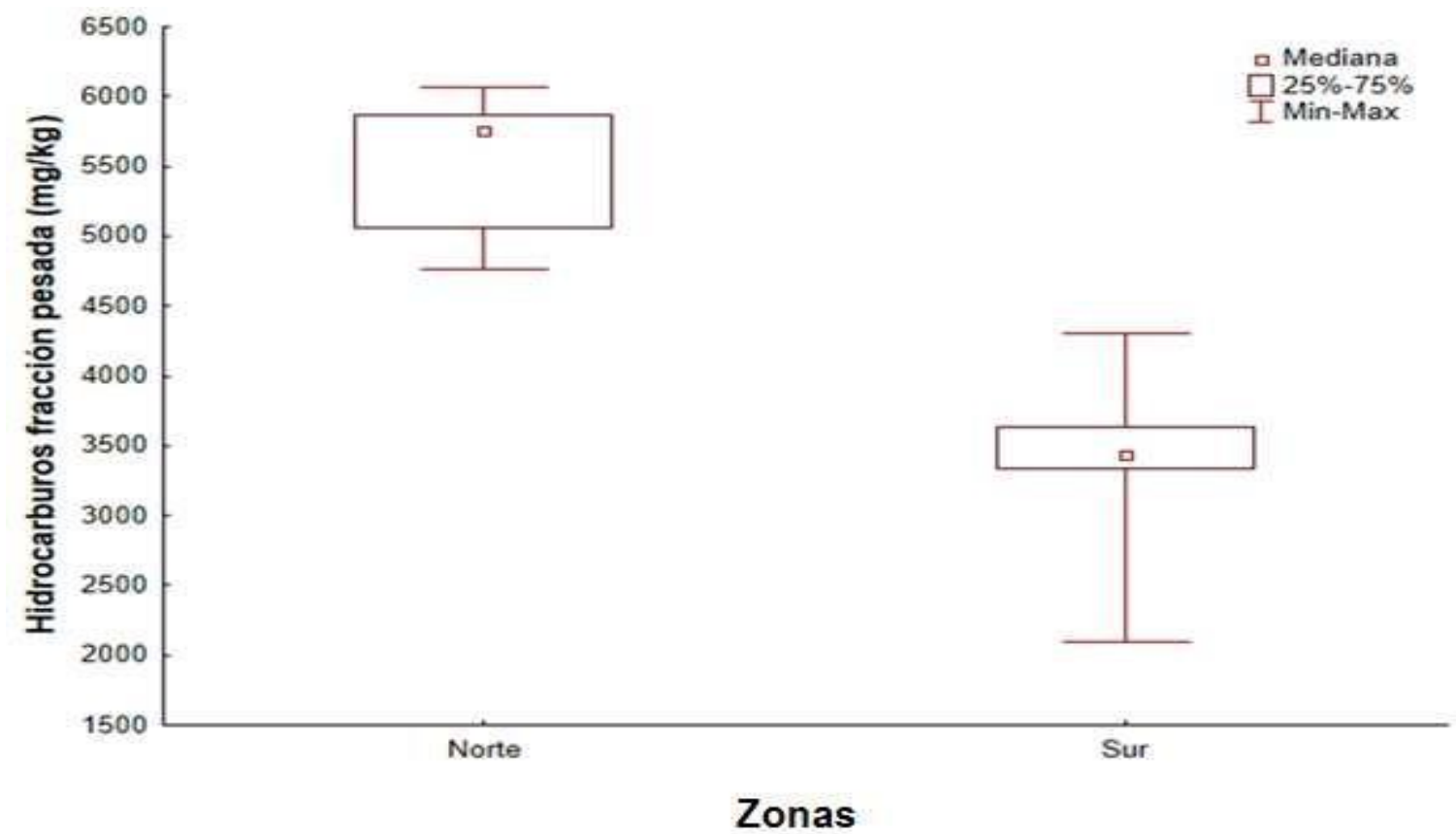

Figura 2. Contenido de Hidrocarburos fracción pesada en dos zonas: ZN y ZS del sitio Ramsar 1602.

\section{DISCUSIÓN}

Es bien conocido que la contaminación ambiental es útil para el monitoreo y entendimiento de la respuesta anatómica y morfológica de plantas, en éste caso, las tres especies de mangle ubicadas en la $\mathrm{ZN}$ presentan deficiencias evidentes de crecimiento y desarrollo. Los efectos negativos por la relación contaminante-individuo han sido explicados en varios estudios, en Campeche los efectos tóxicos de los hidrocarburos se analizaron en plántulas de tres especies (A. germinans, L. racemosa y $R$. mangle), concluyendo que a mayor cantidad y exposición de contaminación disminuyó el diámetro, altura, producción de biomasa de $A$. germinans y $R$. mangle (Requena-Pavón et al., 2012); al sur de Louisiana plántulas de $R$. mangle se trataron con hidrocarburos (aceite $\mathrm{y}$ petróleo crudo), concluyendo que a bajas concentraciones $(<50 \%$ de la superficie) disminuyo el crecimiento lateral del tallo, tallos laterales y número de hojas, y a mayor concentraciones ( $>50 \%$ de la superficie) se redujo la supervivencia (Proffitt y Devlin, 1998); en bosques al norte de Veracruz reportaron disminución clorofila y la estructura general de individuos de A. germinans por fracción pesada de hidrocarburos (Vázquez, 2012); en Acayucan, Veracruz bajo condiciones de vivero, el tiempo de germinación de semillas de $C$. erectus aumento a varias concentraciones de hidrocarburos (Trejo et al., 2011); en Queensland, Australia, la correlación entre hidrocarburos en sedimentos (predominancia de Avicennia) y la aparición de mutantes deficientes en clorofila a fue muy alta, muriendo los individuos a las pocas semanas de la germinación (Duke y Watkinson, 2002).

Lo anterior muestra que la reacción de las especies de manglar ante el daño por hidrocarburos es muy variada ya sea por el tipo de contaminante, composición, tiempo de exposición, o incluso por las características 
particulares tanto de la especie como del sitio donde se localiza. Esta investigación y sus resultados fortalecen la necesidad de continuar indagando sobre los agentes causales que modifican la estructura del Sitio Ramsar 1602 y así, favorecer su conservación desde una perspectiva integral y no de manera aislada; puesto que conocer el contenido de hidrocarburos en zonas protegidas favorece entre otras cosas a dar mejor manejo de la zona afectada para comprender el escaso desarrollo de las especies que habían en ellas, principalmente las especies de manglar.

\section{CONCLUSIONES}

Ambas zonas hay hidrocarburos fracción pesada (HFP), descartando la hipótesis inicial de que en la ZS no había presencia de ellos.

Ambas zonas evaluadas (norte y sur) del Sitio Ramsar 1602, tuvieron concentraciones de HFP por encima del límite máximo permisible para áreas de uso de suelo residencial, forestal, recreativo y/o de conservación.

La zona norte sobrepasó el límite máximo permisible de HFP con $80 \%$.

La mayor presencia de hidrocarburos genera deterioro y eliminación de plantas de mangle.

Se debe analizar de forma puntual, qué tipo de hidrocarburos fracción pesada son los que se encuentran en el Sitio Ramsar 1602.

\section{LITERATURA CITADA}

Basáñez-Muñoz, A. (2005). Ficha informativa de los humedales Ramsar: manglares y humedales de Tuxpan. Facultad de Ciencias Biológicas y Agropecuarias. Universidad Veracruzana. p. 14.
CONABIO. Comisión Nacional para Conocimiento y Uso de la Biodiversidad. (2009). Manglares de México: Extensión y distribución. ( $2^{\mathrm{a}}$ ed.). Comisión Nacional para el Conocimiento y Uso de la Biodiversidad. México. 99 p.

Duke, N. C., Watkinson, A. J. (2002). Chlorophyll-deficient propagules of Avicennia marina and apparent longer term deterioration of mangrove fitness in oil polluted sediments. Marine Pollution Bulletin. 44: 12691276.

https://doi.org/10.1016/Soo25-326X( 02)00221-7

EPA: Environmental protection agency. (1998). Method 9071B N-Hexane extractable material (HEM) for sludge, sediment, and solid samples. USA. p. 13.

EPA: Environmental protection agency. (2000). Method 1664A: N-Hexane Extractable Material (HEM; Oil and Grease) and Silica Gel Treated NHexane Extractable Material (SGTHEM; Non-polar Material) by Extraction and Gravimetry. USA. p. 23.

FAO: Food Agency Organization. (2010). Evaluación de los recursos forestales mundiales 2010. Informe principal. Roma, Italia. p. 346.

Farrapeira, C. M. R., Ramos, C. A. C., Barbosa, D. F., Melo, A. V. O. M., Pinto, S. L., VerÇosa, M. M., OliveiraVeira, D. A. S., Francisco, J. A. (2009). Vertical zonation of the hard substrata macrofauna of the Massangana River 
estuary, Suape Bay (Pernambuco, Brazil). Biota Neotropica, 9 (1): 87-100.

INE: Instituto Nacional de Ecología. (2005). Evaluación preliminar de las tasas de pérdida de superficie de manglar en México. p. 21.

Moreno-Casasola, P., Rojas, J., Zárate, D., Ortiz, M., Lara, A., Saavedra, T. (2002). Diagnóstico de los manglares de Veracruz: distribución, vínculo con los recursos pesqueros y su problemática. Madera y Bosques, Número especial, 61-68. https://doi.org/10.21829/myb.2002.8 01292

Proffitt, E. C., Devlin D. J. (1998). Are there cumulative effects in red mangroves from oil spill during seedling and sapling stages. Ecological Applications, $\quad 8$ : 121-127. https://doi.org/10.1890/1051-0761(19 98)oo8[0121:ATCEIR]2.o.CO;2

Requena-Pavón, G. C., Agraz-Hernández, C. M., Vázquez-Botello, A., Osti-Saénz, J., Reyes-Castellanos, J. E., Chan-Keb,
C. (2012). Efectos del petróleo en la regeneración natural del bosque de mangle: fase experimental. 38-39 p.

SEMARNAT: Secretaría de Medio Ambiente y Recursos Naturales. (2013). NOM138-SEMARNAT/SSA1-2012. Límites máximos permisibles de hidrocarburos en suelos y las especificaciones para su caracterización y remediación. 10 de septiembre de 2013. México.

Trejo, V. V., Carmona, D., Cuevas, D. M. (2011). Germinación de Conocarpus erectus L. (mangle botoncillo) Combretaceae en diferentes suelos contaminado. III Congreso Mexicano de Ecología.

Vázquez, C. G. M. (2012). Efecto del derrame de hidrocarburos sobre una especie de mangle. Tesis de maestría. Instituto de ingeniería. Universidad nacional autónoma de México. 119 p.

Copyright (c) 2018 Arianna Rubi González Sánchez, PabloElorza Martinez, Sara Aida Alarcón Pulido, Maria de la Luz Hernández Sánchez y Alejandra Rodriguez Betancourt

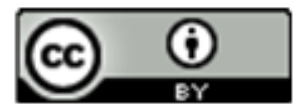

E ste tex to está protegido por una licencia CreativeCommons 4.0

Usted es libre para Compartir —copiar y redistribuir el $\mathrm{m}$ aterial en cualquier medio o formato- y Adaptar el documento - remezclar, transformar y crear a partir del material- para cualquier propósito, incluso para fines comerciales, siempre que cumpla la condición de:

Atribución: Usted debe dar crédito a la obra original de manera adecuada, proporcionar un enlace a la licencia, e in dicar si se han realizado cambios. Puede hacerlo en cualquier forma razonable, pero no de forma tal que sugiera que tiene el apoyo del licenciante o 10 recibe por el uso que hace de la obra.

Resumendelicencia - Textocompletodelalicencia 